\title{
Comparison of Color Doppler Ultrasonography and Clinical Grading of Varicocele in Patients Referred to Shahid Faghihi Hospital in Shiraz
}

\author{
Amin Abolhasani Foroughi, ${ }^{1,}{ }^{*}$ Ehsan Iazdanpanah, ${ }^{1}$ and Ali Ariafar ${ }^{1}$ \\ ${ }^{1}$ Shiraz University of Medical Sciences, Shiraz, Iran \\ "Corresponding author: Amin Abolhasani Foroughi, Shiraz University of Medical Sciences,Shiraz, Iran. E-mail: amin.a.foroughi@gmail.com \\ Received 2016 December 21; Accepted 2017 February 08.
}

\begin{abstract}
Background: Varicocele is one of the most common causes of infertility in men. Although physical examination is the standard method for the diagnosis of varicocele, but in some cases, diagnosis of the disease only by clinical examination is difficult and diagnosis confirmed by ultrasonography. The aim of this study was to compare color doppler ultrasonography and clinical grading of varicocele.

Methods: In this cross-sectional study, 66 patients suspected of varicocele referred to urology clinic of Shahid Faghihi hospital in Shiraz were assessed. After physical examination and clinical grading of varicoceles, the patients underwent color doppler ultrasonography was graded based on Sarteschi system. The collected data were analyzed using SPSS-24 statistical software.

Results: The mean age of patients was $23.16 \pm 6.92$ years. 60 cases $(91 \%)$ had clinical varicocele and 6 cases $(9 \%)$ were healthy and in ultrasonographic assessment, 63 cases (90\%) had varicocele. Clinically, 40 testes were normal(grade0) and 36 testes (27.3\%) had grade 1 of varicocele, 36 testes (27.3\%) grade 2 and 20 testes (15.2\%) had grade 3 and in ultrasound evaluation, 4 testes (3\%) were normal (grade0) and 10 testes (7.6\%) were in grade 1 of varicocele, 26 testes (19.7\%) were in grade 2,41 testes (31.1\%) in grade 3,53 testes (26.5\%) in grade 4 and 16 testes (12.1\%) in grade 5. There was a direct consistency between two systems of clinical and ultrasonographic grading $(\mathrm{P}<0.05)$. Also there was a direct correlation with diameter of varicose veins and clinical grade of varicocele $(<0.05)$. In terms of age, no significant difference in clinical and ultrasonographic grading of varicoceles was observed $(\mathrm{P}=0.36)$.

Conclusions: The results showed that clinical and ultrasonographic grading to diagnose varicoceles is directly consistent together and it is possible to make them equivalent together. In addition, the age factor has not a significant impact on disease grading.
\end{abstract}

This is an abstract presented in the 33rd Iranian congress of radiology (ICR) and the 15th congress of Iranian radiographic science association (IRSA) 